\title{
Commentary
}

\section{What We Need Now}

\section{Breaking It Down and Understanding Words from Mathematical}

\section{Symbols}

\author{
Joseph Martin Stevenson ${ }^{1 *}$ \\ ${ }^{1}$ Joseph Martin Stevenson of Madison, Mississippi is a former provost at Jackson State and Mississippi \\ Valley State Universities. He is currently the Provost for the Global Digital Academy for \\ Class2Class.com, USA
}

Received: July 27, 2020

Accepted: August 3, 2020

Online Published: August 15, 2020

doi:10.22158/jecs.v4n3p77

URL: http://dx.doi.org/10.22158/jecs.v4n3p77

Using words, which is the manifestation of the term "trivium" in the liberal arts of higher education ethos, has been challenging for many of us when discussing inequities embedded, grounded and hidden within the combined forces of today's coronavirus pandemic and disconcerting race relations. Indeed, this has impact, influence, and implications to both education and culture. In recent months, many have experienced challenges to speak certain words of expression as the result of feeling the sense of heighten anxiety, ambiguity, anguish, angst, and even, underlying anger. For most of us, this is the case in some human measure, and these heighten human amplifications and pronounced inner feelings are becoming more and more challenging. There seems to be growing uneasiness with trying to understand the meaning of words like inequality, disparity, justice, prejudice, and lives matter. Since all if these terms can be relative in meaning, perhaps we can leverage the "quadrivium" of the historical seven liberal arts -- arithmetic -- to bring about clarity for interpreting the words through the lens of mathematical symbols. When teaching college students, I refer to this as the phenomenon of "crisscrossing" the liberal arts where the quadrivium meets the trivium at the nexus of finding truth. Could it be that we can find, express, and deepen understanding of truth from and with cross-crossed translations between words and numbers? Is it possible that we could create algorithms to address inequities, disparities, and injustice? In this commentary's case, I am suggesting that we leverage the quadrivium, which is about the use of words, and the trivium, which is about the use of mathematics, to help us understand the power of words and the power of math in our everyday language, lexicon, dialogue, and communications with each other. What I am proposing is a simple, yet complex, way to deepen mindful understanding during these trying times of chaos, confusion, and conflict. Perhaps what we need now are mutual interpretations and 
defining translations to generate general understanding with very specific meaning. A note of caution. I realize many of these assertions are relative, metaphorical, partial, and biased, but the purpose here is to start conversations about balanced revelation of thought, expression, and mindfulness. For example, let us consider the math symbol of = which comes up a lot in our thinking, speaking, writing, and utterance. The $=$ symbol (equal to) is best understood when we realize it is probably not going to happen until and unless we seriously examine and respond to social injustice that is pervasive, permeating, and prevalent just about everywhere in America. Let us address the $\div$ symbol (division). This symbol is certainly referenced a deal in recent years as the result of emerging divisiveness, division, and divide between and among many opposing groups. Just as we take from one domain and give to the other in mathematical division, we must do address and react to social injustice. The + symbol (addition or positive) is central to the type of thinking we must embrace for comprehending the bigger picture, the larger context, and the greater good.

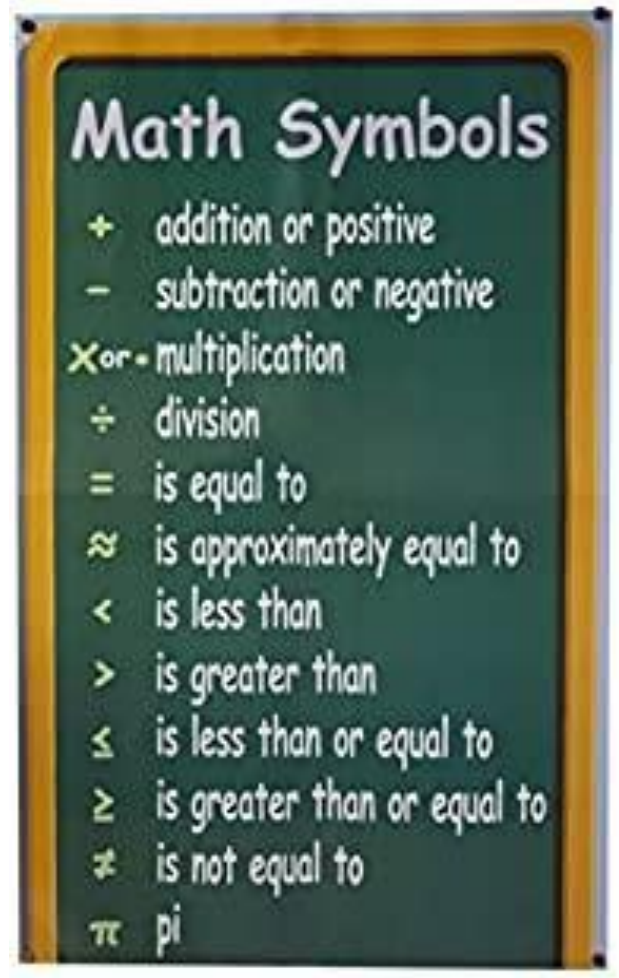

\section{Figure 1. Math Symbols for Social Justice}

In education and culture, we can do more together when we apply these fundamental mathematical symbols, and the potential unification they could represent, all over the world. Afterall, mathematics is the universal language. In contrast, the - symbol, meaning either subtraction or negative is sometimes referred to when describing populations in our society who are marginalized, devalued, voiceless, or experience inequity. The symbol < means "less than". This mathematical symbol can be applied when we make reference to certain populations as underutilized, unrepresented, under-resourced, under-served, or 
underprivileged; whereas the symbol > meaning "greater than" could be applied when referencing comparisons in health disparity, employment disparity, criminal justice disparity, economic disparity, or workforce disparity where some populations are clearly advantaged -- from data and documentation-over others. The symbol $\mathrm{x}$ in mathematics or arithmetic means multiplication. This symbol can describe the overwhelming need for the levels, layers and lifts we will have to advance in order for this country's enslaved, immigrant, native, and indigenous people to reach for future greatness.

Other math symbols are identified below for readers to consider during thoughts and times of need for didactic interpretation, translations, and understanding -- using the quadrivium and trivium of the liberal arts or the left side and right side of the brain. One last symbol for this commentary is "pi" listed last above, which is commonly interpreted as "squaring the circle".

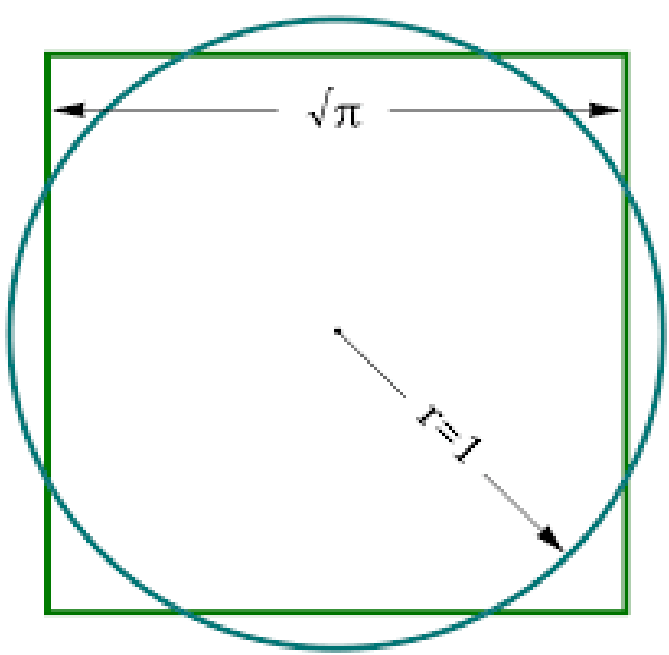

Figure 2. Squaring Circles for Social Justice

It may be that most of us spend too much time in life trying to square the circle of others, but it does not mean we should stop trying to empathize with others and face our past prejudicial biases, our present subtle subjectivity, and our future potential divisive influences. In fact, all us must work on bringing the distance between the lined squares and circles of our human divisiveness. Finally, it would be quite interesting, informative, insightful and, perhaps , inspiring to see how all of us would -- individually and collectively -- define the symbols for "approximate equal to", "less than or equal to", "greater than or equal to" and "not equal to" in this commentary's esoteric, yet, eccentric context about what we need to now to break down language and lexicon barriers of communication and good intentions in the spirit of crisscrossing the historical and still-relevant today's liberal arts, more than ever, as we face and confront the complexities of the opposite definitions of social distancing for our race relations and the coronavirus pandemic. Since most of communication is done electronically, online, virtually, and via the Internet these days in cyberspace, I suggest we begin there with the first stop being social media interactions between and among people. 
Between applying mathematical symbols and squaring circles, we should be able to engage in metaphorical, metaphysical and meta cognitive discussions about understanding the deeper premises of social justice through the wider principles of mathematical science and sense-making. 